\title{
Jalons du développement de la cardiologie actuelle
}

\author{
Roger Hullin ${ }^{a}$, Beat Kaufmann ${ }^{b}$, Michael Kühne ${ }^{c}$, Hans Ricklid, Peter Wenaweser ${ }^{\mathrm{e}}$, Michael Zellweger \\ a Prof., président du groupe de travail Insuffisance cardiaque de la SSC, CHUV Lausanne \\ b Prof., président du groupe de travail Echocardiographie et Cardiac Imaging de la SSC, Hôpital universitaire de Bâle \\ c Dr, privat-docent, membre du groupe de travail Stimulation cardiaque et électrophysiologie de la SSC, Hôpital universitaire de Bâle \\ d Prof., président du Steering Committee AMIS Plus, Hôpital cantonal de Saint-Gall \\ e Prof., président du groupe de travail Cardiologie interventionnelle de la SSC, Klinik im Park, Zurich \\ f Prof., président de la SSC, Hôpital universitaire de Bâle
}

Etant donné l'évolution démographique, les maladies cardiovasculaires touchent de plus en plus de personnes et représentent en Suisse la principale cause de décès. Les hospitalisations dues aux maladies cardiovasculaires ont, à elles seules, concerné 114000 personnes en 2014. Divers exemples permettent d'illustrer les bénéfices de la cardiologie de ces dernières décennies dont ont profité ces personnes et d'autres patients.

Des méthodes plus douces, plus rapides et plus exactes permettent des diagnostics plus précis et des thérapies plus efficaces

Les examens d'échocardiographie ont fortement marqué la médecine cardiovasculaire moderne. Ce qu'un stéthoscope permettait avant de supposer peut aujourd'hui être représenté par une image ultrasonique détaillée et quantifiée - et ce, sans aucune exposition aux rayons: anomalies valvulaires, insuffisance de la fonction de pompage du cœur, p. ex. lors d'un infarctus du myocarde, usure ou infection des valves cardiaques, épanchement péricardique, effets de maladies systémiques sur le cœur. Ce diagnostic amélioré et rapidement disponible dans les situations d'urgence est devenu incontournable dans la cardiologie moderne. Les nouvelles thérapies douces utilisées pour traiter les valvulopathies cardiaques, qui profitent à une popu-

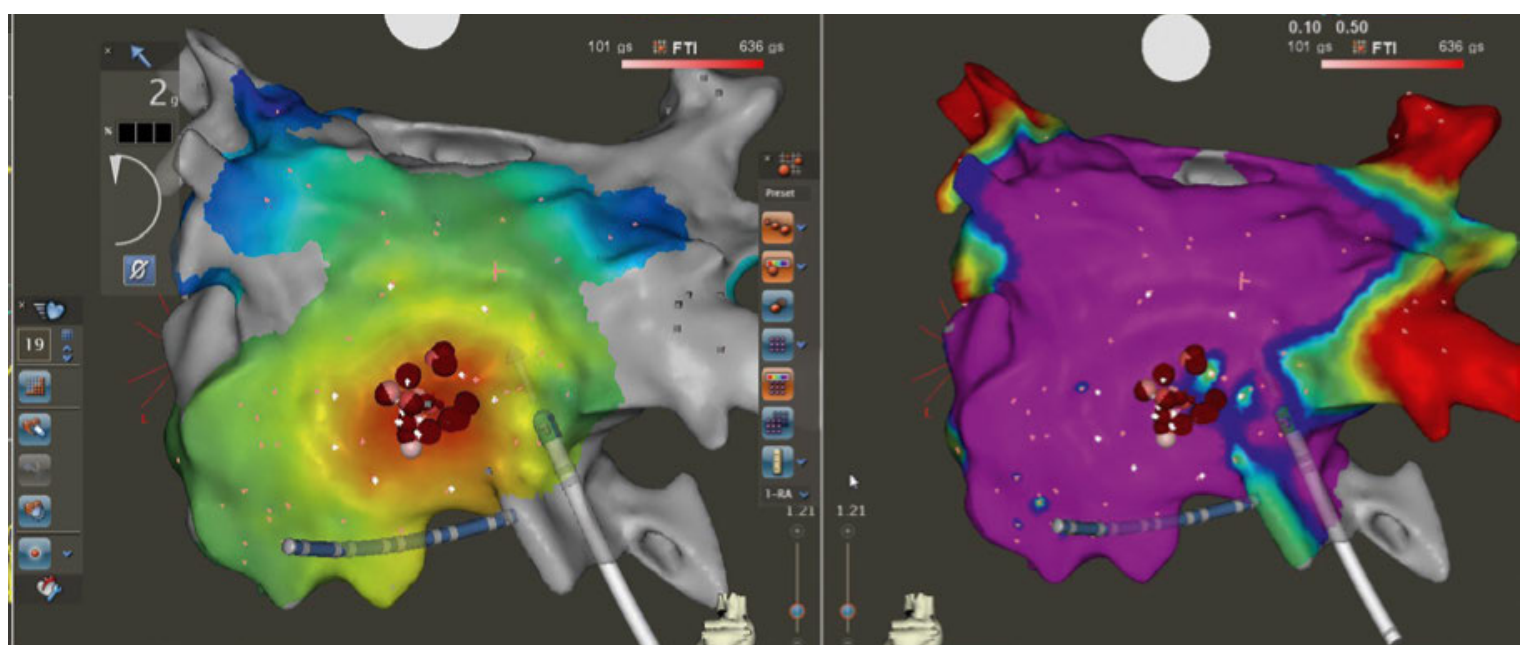

La cardiologie s'est fortement développée ces dernières décennies - au bénéfice de nombreux patients. Grâce à des méthodes telles que I'ablation par cathéter (=sclérothérapie), les patients souffrant de troubles les plus divers du rythme cardiaque peuvent aujourd'hui être traités et même, dans de nombreux cas, durablement guéris. Les images montrent la reconstruction tridimensionnelle d'une oreillette gauche (de derrière) avec le cathéter d'ablation (en blanc) et un cathéter de diagnostic (en bleu). Les points rouges indiquent l'endroit où la tachycardie a pu être terminée avec l'ablation. Les reconstructions permettent d'exécuter l'examen de facto sans rayons. 
lation toujours plus vieillissante, ne seraient pas réalisables sans l'imagerie par ultrasons complexe et en partie tridimensionnelle.

Les techniques d'images en coupe transversale telles que l'IRM du cœur (tomographie par résonance magnétique), la tomographie par ordinateur, la scintigraphie et la tomographie par émission de positons (TEP), permettent une application axée sur le patient et la problématique. Grâce à ces méthodes, il est possible de détecter ou d'exclure de manière très fiable un problème au niveau des artères coronaires. L'IRM et les méthodes de médecine nucléaire permettent de caractériser le tissu cardiaque afin de déceler des inflammations ou des dépôts, là où auparavant un échantillon de tissu était nécessaire. Ces techniques permettent la planification et la réalisation exactes d'interventions au niveau du cœur et des valves cardiaques beaucoup moins éprouvantes pour le patient qu'il y a encore quelques années.

\section{De nombreux troubles du rythme cardiaque sont devenus curables ces 20 dernières années}

Madame Anderegg (nom modifié) est l'une des nombreuses patientes traitées ces dernières années dans un centre suisse pour arythmie cardiaque. Malgré un traitement médicamenteux, elle souffrait depuis des mois d'épisodes réguliers de tachycardie. Ses absences répétées dues à son arythmie cardiaque lui ont fait perdre son emploi de vendeuse. Jusqu'au début des années nonante, il n'existait aucune mesure médicale susceptible de soulager cette patiente. Aujourd'hui, une sclérothérapie (ablation par cathéter) est une aide précieuse qui a permis de guérir les troubles de rythme cardiaque de la patiente. Cette dernière a elle-même été surprise de la rapidité avec laquelle elle a retrouvé une vie normale. Elle travaille aujourd'hui à nouveau à plein temps.

Les symptômes de l'arythmie cardiaque qui touche en Suisse des milliers de personnes sont multiples: la tachycardie et les malaises sont fréquents, les crises d'étouffement et la perte de connaissance occasionnelles. Tandis que les électrochocs et les médicaments étaient pratiquement les seules thérapies disponibles auparavant - thérapies souvent inefficaces qui ne traitaient que les symptômes sans s'attaquer aux causes de la maladie -, des méthodes (ablation par cathéter = sclérothérapie) ont été développées ces 20 dernières années qui permettent aujourd'hui de traiter les patients souffrant des troubles les plus divers du rythme cardiaque et même, dans certains cas, de les guérir durablement. En 2016, 6431 sclérothérapies ont été réalisées en Suisse. Ces interventions exigent une infrastructure hospitalière spécifique de pointe. Grâce au recours supplémentaire à la visualisation tridimensionnelle fournie par les ultrasons ou l'IRM, ces interventions présentent désormais très peu de risques ( $<1-2 \%$ de complications graves).

Lorsqu'une arythmie cardiaque dangereuse, voire une fibrillation ventriculaire apparaît, il est aujourd'hui possible d'implanter au patient un défibrillateur «miniature». Le défibrillateur implantable (ICD) a fait son apparition dans le quotidien clinique il y a une vingtaine d'années. Aujourd'hui, l'opération est devenue une intervention de routine grâce aux améliorations continues et sert pratiquement d'ange gardien aux patients présentant un risque accru d'arythmie dangereuse puisqu'elle est en mesure de traiter de manière efficace des troubles du rythme cardiaque potentiellement mortels.

\section{Accéder par les vaisseaux - des interven- tions moins risquées ouvrent des options thérapeutiques à plus de patients}

Cette année, la cardiologie interventionnelle fête les 40 ans de la première dilatation au ballonnet par A.Grüntzig. Cette technique qui permet d'élargir des artères coronaires rétrécies ou obstruées à l'aide d'un cathéter à ballonnet gonflable sauve la vie à de nombreux patients lors d'un infarctus ou leur épargne un pontage coronarien au stade chronique. Après cette invention révolutionnaire dans le traitement des maladies coronaires, d'autres avancées importantes ont été réalisées. Des stents coronaires ont permis de soutenir durablement les vaisseaux et d'en prévenir un (nouveau) rétrécissement. Par ailleurs, grâce aux médicaments hypolipidémiants, de nombreuses maladies cardiovasculaires ont pu être évitées. Ces facteurs contribuent, parmi de nombreux autres, à l'espérance de vie de la population suisse qui est l'une des plus élevées au monde. Au cours des 25 dernières années, l'espérance de vie en Suisse a augmenté de 6,7 ans chez les hommes et de 4,1 ans chez les femmes.

Pendant des années, on a sous-estimé l'impact des valvulopathies - soit des maladies des valves cardiaques sur l'espérance mais aussi la qualité de vie. Une grande partie des patients âgés souffrant de valvulopathies graves ont été traités de manière palliative et non par la chirurgie cardiaque conventionnelle à cause du risque trop élevé que présentait une opération à cœur ouvert. La première implantation minimal-invasive d'une valve aortique (TAVI) en 2002 a été une avancée décisive dans le traitement des malformations cardiaques. Ce traitement efficace s'est rapidement établi en Suisse aussi où il occupe, après la dilatation au ballonnet, la deuxième place des interventions les plus 
fréquentes dans la cardiologie interventionnelle (1630 patients en ont bénéficié en 2016). L'impact sur le bien-être et l'espérance de vie des patients est positif et rend l'intervention particulièrement attrayante grâce à son invasivité minimale. Parallèlement, des méthodes minimal-invasives ont également été développées au cours des dernières années en vue de la plastie ou du remplacement de la valve mitrale qui chez les personnes en bonne santé - empêche le reflux $\mathrm{du}$ sang depuis le ventricule gauche vers l'oreillette gauche. Ces techniques ouvrent de précieuses options thérapeutiques (p. ex. MitraClip) aux patients auparavant inopérables ou à haut risque souffrant d'une insuffisance mitrale grave. Ces avancées sont le résultat de l'étroite collaboration entre cardiologues et chirurgiens cardiaques.

\section{La mortalité de l'infarctus a nettement diminué}

Le diagnostic et le traitement de l'infarctus du myocarde ont considérablement évolué au cours des 20 dernières années. Il y a 40 ans, on prescrivait plusieurs semaines de repos aux patients ayant subi un infarctus. Aujourd'hui, on peut agir activement contre ses conséquences.

Le Registre AMIS-Plus qui regroupe plus de 50000 patients et qui s'est établi depuis 1997 dans les hôpitaux suisses montre que l'introduction notamment de l'intervention coronarienne percutanée aiguë (PCI aiguë) associée à des mesures médicamenteuses améliorées a permis de réduire fortement le nombre de décès dûs à un syndrome coronarien aigu chez les patients hospitalisés: chez les hommes, la mortalité a diminué de 9,8 à 5,5\%, et chez les femmes même de 18,3 à $6,9 \%$. Le registre fournit une multitude de données épidémiologiques et permet aux hôpitaux d'optimiser leur assurance qualité, notamment grâce à un benchmark national. La mortalité de l'infarctus du myocarde en Suisse est l'une des plus faibles grâce à une prise en charge pluridisciplinaire toujours plus performante des patients concernés.

\section{Le traitement de l'insuffisance cardiaque n'a jamais été aussi efficace}

L'insuffisance cardiaque décrit une faiblesse du cœur avec des répercussions sur l'ensemble de l'organisme. Lorsque la capacité de pompage du cœur diminue, le patient souffre de détresse respiratoire, d'œdème pulmonaire, de rétention d'eau dans le corps entraînant une détérioration d'autres organes. L'insuffisance cardiaque est une maladie très répandue qui touche 1,5 à 2,5\% de la population des pays occidentaux et quelque 175000 personnes en Suisse. La maladie est rare chez les personnes jeunes, mais touche déjà $2 \%$ des 55-64 ans et jusqu'à 20\% des personnes très âgées. Avec le vieillissement de la population, le nombre de patients souffrant d'insuffisance cardiaque devrait doubler dans les 20 prochaines années.

Une recherche clinique intensive a fortement amélioré les possibilités de traitement de l'insuffisance cardiaque au cours des trois dernières décennies. On dispose aujourd'hui de diverses classes de médicaments qui permettent de diminuer les troubles quotidiens et les limitations des patients souffrant d'une insuffisance cardiaque ainsi que leur taux de mortalité. Par ailleurs, des stimulateurs cardiaques spécifiques permettent d'améliorer la fonction de pompage du cœur chez certains d'entre eux. Une guérison reste toutefois rare et l'évolution de la maladie n'est en général qu'une question de temps. Néanmoins, les patients concernés bénéficient d'une vie prolongée de meilleure qualité avec moins d'hospitalisations et peuvent mener une vie (plus) autonome.

Les exemples cités ici montrent une "explosion des avancées» en cardiologie qui ouvre au grand nombre de patients malades du cœur des possibilités et des perspectives qui étaient encore impensables il y a 20 ans. Cette évolution est le résultat d'une recherche continue, de la formation prégraduée, postgraduée et continue, d'un dur labeur, de progrès technologiques et d'un système de santé de haute qualité et accessible à tous.

\section{Résumé}

"Vieillir, c'est aussi se bonifier». Cette citation de Jack Nicholson caractérise parfaitement les avancées en cardiologie. Ces dernières années, cette discipline a connu un développement spectaculaire et engendré des bénéfices pour nos patients. Des méthodes d'imagerie permettent de poser rapidement des diagnostics précis. Nos patients bénéficient ainsi d'un conseil et d'un traitement efficaces. Des interventions d'urgence dans le but de dilater les artères coronaires lors d'un infarctus ont considérablement réduit la mortalité. De nouveaux médicaments et appareils tels que les stimulateurs cardiaques permettent de remédier avec succès aux problèmes à long terme de l'insuffisance cardiaque et à la souffrance des patients. Ces avancées impliquent une formation postgraduée et continue de haute qualité du corps médical engagé en cardiologie et des équipes pluridisciplinaires impliquées.

Crédit illustration

๑) Service de cardiologie, Hôpital universitaire, Bâle 